\title{
2 Altered, Expanded and Distributed Embodiment: the Three Stages of Interactive Presence
}

\author{
John A. Waterworth and Eva Lindh Waterworth
}

\begin{abstract}
This conceptual chapter outlines three stages in the development of interactive presence, and outlines some possibilities and challenges raised by each, and by their combination. The first stage, presence via altered embodiment, refers to the way technology allows us to experience the world with modified or enhanced senses. The second stage, via expanded embodiment, refers to technology pushing the envelope of the mental body in which one feels present, out beyond the physical body. Finally, distributed embodiment refers to how the sense of being present in the world can be separated from that of ownership of a particular body, through the development of new approaches to deploying the technologies of virtual realization. We suggest that presence is the yardstick of embodiment from an experiential perspective. If you cannot feel presence, you are not embodied in the world.
\end{abstract}

Keywords: Embodiment; Perception; Augmented Reality; Virtual Reality; Nonmediation.

\subsection{Introduction to Chapter 2: Presence and Three Categories of Embodiment}

We define presence as the feeling of being bodily located in a perceived external world. This definition applies to both unmediated and mediated presence. Experienced variations in the strength of this feeling provide vital information to the organism in its struggle for survival (see Waterworth et al., 2010 for more details). A useful definition of presence must have implications for what is not presence (Floridi, 2004) and we have previously termed this "absence", a state of absorption in the internal world of the mind detached from the current perceptual flow (Waterworth \& Waterworth 2001, 2003). Our view of presence is thus closely related to embodiment in the external world and to attention. Indeed, we see the possibility to feel present (in the present time, in a present place) as a defining characteristic of embodiment. In the same way, the possibility to experience presence in an external reality mediated by technology is a defining characteristic of embodiment in interactive media.

When we feel present in the physical world, we are naturally embodied as the human beings we are. We have a certain kind of body, acting according to certain perceptual and motor capacities that allow us to move and act in the world. We also have a sense of our own bodies, as part of our self-consciousness, which may be more or less salient at a given time. But experiencing presence is not the same thing as being embodied. When we experience presence our attention is directed towards the 
world in which we find ourselves. Often we are attending to something else, such as thoughts, memories or plans, and then we do not feel much presence, we are mentally absent from the current world around us.

We are seldom very aware of "the technology" making possible our experience of being in the world unless there is something wrong with it, such as when we feel ill or are injured. Rather, the human bodily "technology" delivers the possibility of an experience of being present as a self, located in a body, and surrounded by a world that is not part of us - but only when we attend to that world.

In the same way, mediated presence is possible only via technology that we are not currently aware of as having that role (Biocca, 1997; IJsselsteijn, 2005), giving us a perceptual "illusion of non-mediation" (Lombard and Ditton, 1997) - which is a popular definition of presence. In that case, our attention may be directed to the present mediated reality in which we have (at least to some extent) the illusion of being physically located. In the cinema, for example, if we are highly aware of the projector, the sound system, the seating, and so on, we are unlikely to feel much sense of presence in the movie we are watching. Instead, the physical world of the theatre will be where we experience being present.

The experience of presence in a computer-mediated environment is also often a function of the possibilities for direct action in that world, in the same way that feeling present in the physical world is grounded in perception and bodily action. Unless we can act in a world (whether physical, virtual, or a mixture of both) we will not feel ourselves to be greatly present in it. When we act in mediated worlds, we may interact through different degrees and forms of embodiment and this has implications for the extent to which we can potentially feel present in the world (Haans \& IJsselsteijn, 2012). We suggest that in principle we can understand when and how some technologies become embodied and others do not by examining the strength and nature of our feelings of presence in the world the technology creates or mediates.

The three categories of embodiment we will describe in the rest of the chapter represent progressive stages in the design of interactive presence. They are key concepts for understanding possible ways in which designed interactive experiences affect and will continue to change our experience of the world and of our own bodies in the future. They are stages both in a progressive sense and in terms of their distance from the physical body.

The first approach, presence via altered embodiment, refers to the way technology allows us to experience the world with modified or enhanced senses. If one hears (what would naturally be experienced as) sights and looks at (what would naturally be experienced as) sounds, what becomes of one's sense of being present in the world, of one's bodily relation to it? The second approach, via expanded embodiment, refers to technology pushing the envelope of the mental body in which one feels present, out beyond the physical body. This involves the incorporation of information technology as part of the self, implying a change in the boundary between the self and the non-self (the other) that constitutes the world around one. Finally, distributed 
embodiment refers to how the sense of being present in the world can be separated from that of ownership of a particular body, through the development of relatively new approaches to deploying the technologies of virtual realization.

In the following sections, each of these three categories of interactive presence is described, followed by a consideration of various possibilities and challenges raised by them, and by their combination.

\subsection{Altered Embodiment: Changing Senses, Changing the World}

Don Idhe (1990) distinguished between an embodiment relation between a person and a technological artefact and a hermeneutic relation. In the former, the technology becomes embodied - as if part of the user's body - and perception is through the technology, which itself is essentially transparent. In the latter, in contrast, the user must interpret a more abstract representation of the information the technology provides. For us, Ihde's embodiment relation is a clear example of altered embodiment, which is characterized by a situation whereby my viewpoint is located in my physical body as normal, but I have changed perception, so that my sense of presence in the world around my body is potentially altered in some way and the technology mediates a world of which it is not perceived to be a part.

Altered embodiment is often an intended or unintended aspect of interacting with digital technology. Virtual Reality (VR) can, in some circumstances, achieve a kind of "sensory rearrangement" resulting in modified experiences of one's own body (Biocca and Rolland 1998; Castiello et al. 2004; Normand et al. 2011; Riva 1998; Riva et al. 2011). In addition, altered embodiment can be seen as part of a general trend in the way many computer applications are designed: as perceptual tools, rather than cognitive artefacts (Norman, 1993). Waterworth (1997) refers to the potential of "synaesthetic media" - computer applications that provide an experience of information that is usually perceived in one form in a radically different form - to support enhanced creativity through new ways of perceiving information (see also Waterworth, 2003).

When the idea of synaesthetic media is applied to a body functioning normally in the physical world, technology becomes a perceptual transducer producing a radically altered state of embodiment in the world. As an example, imagine how it would feel to have your senses rewired. This is the experience of wearing the Reality Helmet (Waterworth and Fällman, 2003), a wearable computerised system developed with the purpose of providing its users with altered interactive experiences of physical reality. It consists of a custom-made helmet that the user wears, and computational equipment placed in a custom built backpack, which allows a high degree of mobility for its wearer (Figure 2.1). The eyes and ears are completely covered, so that users become audio-visually isolated from the environment, while the other senses are not interfered with. A digital video camera and stereo microphones are mounted on the outside of the helmet, while inside there are a pair of small visual displays and 


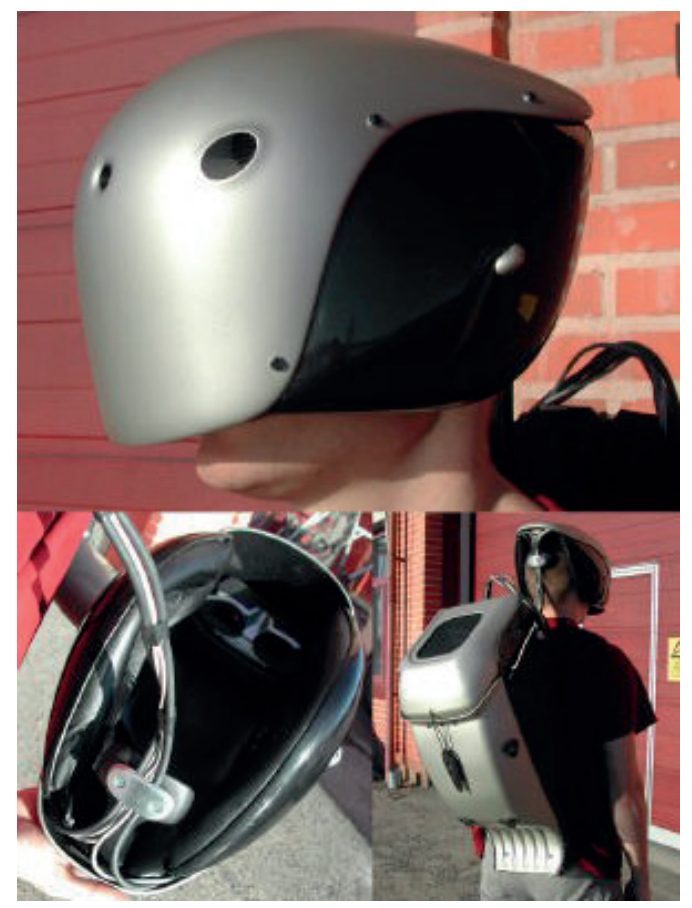

Figure 2.1: The Physical Set-up of the Reality Helmet

headphones. Through computer signal processing, the user's perceptual experience is transformed by providing a real-time visualization of the auditory environment in which the wearer is situated and, likewise, a landscape of sound generated from digital video input. The user sees what she would normally hear, and hears what she would normally see.

Applications such as the Reality Helmet challenge the argument that degree of presence is a simple function of the level of subjective realism (see also Bouchard et al., 2012), at least in the normal sense of the word. A user trying to navigate the physical world while wearing the helmet will feel highly present, because almost all her attention will be directed towards perceptual information - much of which will be provided to her via the helmet and associated technology. Navigation of the world is not easy, because of the novelty of this form of perception. But the technology does effectively become part of the body - an altered body with changed senses applied to the physical world.

In altering the nature of embodiment, the form of the world is also changed. We naturally take for granted that the world has the form we normally perceive it to have, even though we may know that the senses we have are different from the senses other animals have, and that their perceptions of the world will be different from our own. The physical world exists and has real content - this is the actual environment in 
which organisms strive to survive - but its form is a matter of perception. Evolutionary adaptation results in forms of perception for particular types of organism (people, bats, cockroaches) that have tended to help them survive over many generations. Through the technical mediation that characterises altered embodiment, we can now choose other forms - of perception and therefore of the world - that may help us to function in the rapidly changing mediated world we humans now inhabit.

The Reality Helmet usefully exemplifies the notion of presence through altered embodiment and is unusual, possibly unique in the way two major senses are 'reversed' to completely alter how external reality is perceived. But there are many other examples of the related idea of sensory substitution (e.g., Bach-y-Rita \& Kercel, 2003 is a well-known example), most commonly developed as assistive technology for those with some sensory disability such as blindness. "The vOICe" (The vOICe, 2012) is a recent system providing "augmented reality for the totally blind". Its main functionality is to convert video camera images into sound to enable the blind to navigate the world (and other information) by hearing instead of seeing.

Altered embodiment is the first stage of interactive presence. When our embodiment is altered and we feel present in the physical world that surrounds the body, our perceptions of that world are radically changed. At the same time, when this is achieved to a high degree, we experience a highly effective perceptual illusion of non-mediation, even though the world is dramatically changed for us relative to our everyday experience.

\subsection{Expanded Embodiment: Embodiment without a Body?}

Some degree of expanded embodiment accompanies mediated presence as the term is commonly understood (Bracken \& Skalski, 2010). This is most clear in a VR environment where the actual physical surroundings are shielded from the user as far as possible - to avoid distraction away from the virtual world. These distractions have been termed "breaks in presence" (Slater \& Steed, 2000) but are actually shifts of presence from the virtual world to in the physical world.

As with our sense of presence in the physical world, a first-person perspective is often a key ingredient in evoking strong presence in media. This is the norm in 'classical' virtual reality, where we view the mediated world as if embodied there ourselves (to some degree) with normal senses and with a first person perspective on things. We move our physical head and the virtual view changes accordingly; we move our physical arms and hands and we see a representation of these body parts depicted as if they were co-located with the internal image we have of our physical body.

Expanded embodiment brings with it the possibility for presence in a mediated world, experienced as a more or less convincing perceptual "illusion of non-mediation" (Lombard \& Ditton 1997). This might be a fictional world, such as an immersive VR game, or the convincing experience of being in another physical place - the original 
goal of telepresence (Minsky, 1980). Presence mediated in this way is the feeling of being embodied in a non-physically-present external world, in the realization of which technology plays an active and direct role. The more the technology disappears from a person's attention and becomes experientially part of the self, the higher the level of presence through expanded embodiment. When this kind of VR realisation is technically done well, there is no conflict between the mediated reality and the user's body schema or body image.

We will not discuss this variety of mediated presence further here, which is not the main focus of this chapter and is the topic of several chapters in this volume (see especially Chapter 1 by Riva and Mantovani). The reader is also referred to our earlier publications on this topic, in particular Waterworth \& Waterworth (2001), Riva et al. (2004), and Waterworth et al. (2010). In the rest of this section, we take the opportunity to discuss other candidates for realising presence through a different form of expanded embodiment.

As already stated, we consider mediated presence to be basically an interactive perceptual illusion (Waterworth \& Waterworth, 2003; Waterworth et al. 2010). It involves more than just perception and action, since high levels of presence cannot be maintained without intellectual and/or emotional engagement, but perception of an apparently real interactive environment surrounding the self is its core. Perception as a process results in hypotheses about what things exist in the immediate environment and what is happening (Gregory 1997), experienced as those things and events. Virtual reality, especially high quality and fully immersive VR with rich interactivity, fools the brain into perceiving that the body is somewhere it physically is not. This is why VR can have such powerful effects on the perceiver (Waterworth et al. 2010).

But high quality VR is expensive and cumbersome and requires that the person who is the perceiver to have normal abilities to act in the world, making it unusable by people who cannot move their bodies in normal ways - such as paraplegics. This latter consideration has helped to drive a line of research and development aimed at achieving direct brain-computer interaction (BCI), whereby a user can interact with a virtual world without moving body parts. Instead, brain activity is captured and interpreted by the computer to carry out intended actions in virtual space. BCI has been successful (e.g., Leeb et al. 2007), but the level of presence achieved is relatively low, as indicated by the significant effort of carrying out actions in the world. A degree of expanded embodiment is achieved however, and motivated users can learn to produce reliably recognised patterns of electrical activity in their brains, sufficient to carry out their intended actions successfully (Hochberg et al. 2006).

In the other direction, computer-brain interaction (CBI) seeks to sidestep perception by presenting a virtual world directly to the brain, by stimulating it with magnetic fields - a technique known as Transcranial Magnetic Stimulation (TMS). The theory is that if this is done in the right way at the correct locations, the necessary electrical activity can be induced in the brain corresponding to the required perceptions of the virtual world. TMS has also been used in psychotherapy 
in the treatment of neurological and psychiatric disorders, such as depression and auditory hallucinations, but the evidence for its effectiveness is weak (Slotema et al. 2010). Interestingly, TMS has become notorious because of its ability to induce a feeling of the presence of another being in the vicinity of the observer (Persinger et al. 2010). Sometimes this is experienced as a divine presence, sometimes a malevolent one, and sometimes as distortions and extensions of the observer's body, while in some studies, an absence of reliable effect has been reported (e.g., Larsson et al. 2005).

In summary, feelings of embodiment can be produced through BCI and CBI, without engaging the bodily systems of action and perception. But these are relatively weak via BCI, because of the effort of action in the virtual world. With CBI, presence effects can be experienced, but may be highly variable or non-existent. In the some cases at least, they produce effects resembling our third step in interactive presence, distributed embodiment.

\subsection{Distributed Embodiment: That's Me Over There!}

According to Metzinger (2006) there are three distinguishable aspects of human embodiment in the world. Like all animals, we are a body with certain physical characteristics and so have what Metzinger terms first-order embodiment. And like all but the simplest animals, we also have associated and integrated perceptual and motor systems that allow our bodies to function effectively in the world, often without the need for attention or even conscious supervision. This 'body schema' comprises Metzinger's second-order embodiment. The third order of embodiment is the 'body image', the mental representation we have of our own bodies and which, it is said, few animals possess.

The sense of what is self and what is not is actually quite flexible, and may be altered to extend beyond the reality of the biological body (e.g., Normand et al. 2009). Some technology can change the boundary of the body, by becoming perceptually part of the self - the blind man's stick is the classic example from phenomenology (Merleau-Ponty 1962) - when it is incorporated into the body schema. When this happens, it is as if the technology were functionally part of the body. When using the technology, it is as if the world starts where the tool ends. The technology is effectively part of the body during use, not of the world in which the body acts.

When we are aware of being in that external world and are not aware of the technology mediating our experience, this produces a feeling of presence through a perceptual "illusion of non-mediation" (Lombard and Ditton, 1997). Haans \& IJsselsteijn (2012) consider third-order embodiment to be necessary for presence, but this is only the case when there is a conflict between the mediated view of the body and the body image of the perceiver. When this happens, the relevant mediating body extension becomes an object in the world rather than an integral part of the 
self. In contrast, in classic perceptual effects such as 'the rubber hand illusion' (Botvinick \& Cohen 1998), the mediated body part (the rubber hand) is integrated with the viewer's body image, to dramatic effect. This and similar effects have been reproduced successfully in virtual reality and mixed reality situations (IJsselsteijn et al. 2006: Holmes N., \& C. Spence. 2007, Slater et al. 2008).

Distributed embodiment goes beyond these cases, by separating the observer from the observed body. By this, we do not refer to the possibility merely to observe oneself as a controllable avatar in a virtual world, even though the characteristics of a represented avatar may have a significant effect on self-perception and behaviour (e.g., the Proteus Effect; Yee \& Bailenson, 2007). An avatar may differ completely from one's own appearance, and even from the way one's actions are mapped onto the bodily responses of the avatar (for detailed coverage of this topic see see Chapter 5 in this volume, by Won et al.).

What we call distributed embodiment is experientially very similar to naturally occurring out-of-the-body experiences (Blackmore, 1984). In these we may feel present, while at the same time observing our bodies from a "disembodied" viewpoint (see Figure 2.2). These are relatively rare in nature, and poorly understood, so that they often connote something supernatural or mystical. The idea of distributed embodiment is stranger and harder to grasp than the other steps in interactive presence that we have discussed, because it is a contradictory state arising from conditions that - as far as the perceiving organism is concerned - should not be possible.

It is already possible to produce the feeling of being in a virtual body that is also experienced as remotely located, separated from our own body (see also Chapter 4 for a broader discussion). Simple technology has been used in this way to produce something similar to out-of the-body experiences for several years (e.g., Ehrsson 2007; Lenggenhager et al. 2007, Petkova and Ehrsson 2008). This is achieved by combining

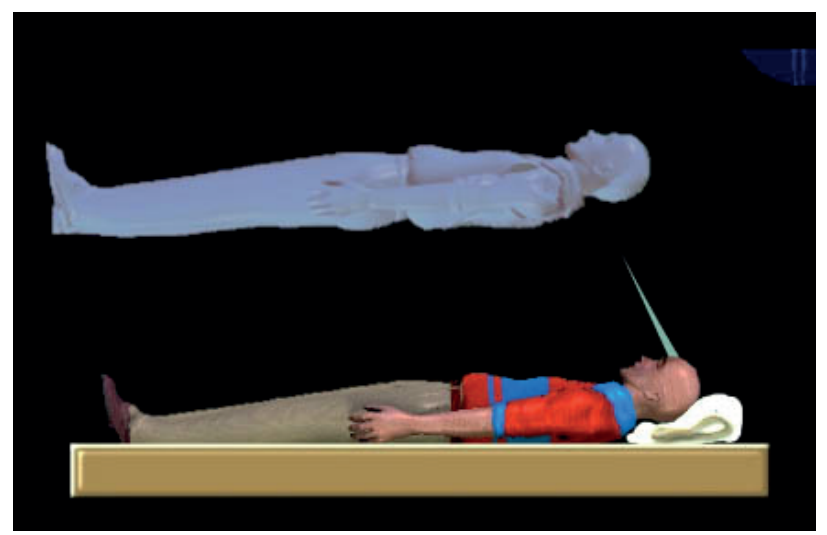

Figure 2.2: Artist's impression of an out-of-body experience. (Image in the public domain) 
tactile and visual stimulation of the observer with corresponding and synchronised displays of the observed body apparently experiencing matching stimulation. As Petkova and Ehrsson report:

Manipulation of the visual perspective, in combination with the receipt of correlated multi-sensory information from the body was sufficient to trigger the illusion that another person's body or an artificial body was one's own. This effect was so strong that people could experience being in another person's body when facing their own body and shaking hands with it. Our results are of fundamental importance because they identify the perceptual processes that produce the feeling of ownership of one's body. (Petkova and Ehrsson 2008, from the abstract)

In these experiments, observers perceive representations of themselves from an external viewpoint while simultaneously experiencing presence as the observed person. The distant body may look like one's normal body, or like someone else. If the latter, the observer would feel present in that person's body, an effect that has already been confirmed through physiologic responses indicating appropriate emotional changes in the observer (see New Scientist 2010; Slater et al. 2010). Ehrsson \& Petkova (2008) suggest that it can be difficult to distinguish actual body transference from more cognitive identification with a representation of their body. But this is a familiar problem (for example in interpreting presence questionnaire responses). In principle, comparison of measures of presence taken on multiple dimensions would enable the two to be distinguished.

\subsection{Our Future Embodiment(s): Challenges and Possibilities}

Many existing computing applications can be viewed as synaesthetic media; they are too numerous and too familiar to be reviewed in detail here. Obvious examples include programs that take an input - such as a stream of music - and turn it into an output in another form - such as a dynamic visual display of the amplitude of various frequency bands. The point is that almost any computer-produced visualization, sonification, haptic or other display that presents information through a realization form other than the original can be conceived of as a synaesthetic medium. But most of these do not provide altered embodiment, because the experience is not integrated with that of the surrounding environment.

Whenever computer-based information is blended with the perception of the surrounding physical world, as in augmented reality this may become integrated into a new form of altered embodiment. But that requires that the augmentation of the physical with the virtual be carried out in such a way that the user has the potential to feel present. Given the clear popularity of mobility and social connectivity, it seems that presence will increasingly be experienced through attention to an external world in which the physical and the virtual are somehow blended (see also Benyon, 2012; Hoshi, Öberg and Nyberg, 2011). This trend is presaged by developments such 
as Google Glass (2013). For this to work in practice, a major challenge is to make media devices sensitive to the situational context of their use, and the state of their users. Presence levels could in principle then be dynamically adjustable to maintain optimal functioning in an unfolding blended reality stream. If this can be achieved, then true Ambient Intelligence (see, e.g., MIT Project Oxygen) might become a reality irrespective of personal location.

The design space of sensory transformations with technology is huge, which provides enormous potential but is also highly challenging for designers. We can see that altered embodiment opens up a new way of being in a world, and of experiencing presence. Indeed, it changes not only the body but also the perceived form of the physical world in which the body is located. In designing altered embodiment, the possibilities are almost endless - but we do not yet know much about what will work best for which purpose, or about possible longer term effects on the perceiver.

Many convincing demonstrations of expanded embodiment already exist, for example in psychotherapy, entertainment, training, and mental and physical rehabilitation. The power of expanded embodiment as produced by well-designed immersive VR applications is already well know, and several of these are treated in detail elsewhere in this volume.

New design possibilities are opened up when already successful approaches to eliciting expanded embodiment are combined with the huge, but relatively untapped, potential of altered embodiment. For example, the most promising potentials for BCI are perhaps in blends of the physical world and the virtual.

Currently available and emerging technologies, especially gaming and teleconferencing systems based on cheap technology (such as Microsoft's Kinect interface for the Xbox gaming platform) can be adapted to locate and track several individual bodies, voices, and faces in a three-dimensional physical space. A robot or avatar could, for example, be programmed to mirror the movements and facial expressions of the person. When combined with synchronised dual sensory stimulation, this opens up a wide range of possible applications - including copresence systems for teleconferencing, entertainment applications such as games or movies, physiotherapy and sports training, and empathy training. An understanding of how and to what extent we can experience real presence in other bodies will be enormously important in these and other fields.

Design challenges of distributed embodiment include specifying the means of being in other bodies, of switching between bodies, and the characteristics of those bodies - which could include human (self or not, lifelike or not), robotic, animal (Nagel, 1974), or even inanimate objects (Misselhorn, 2009). It is likely that not all these possibilities will be effective in practice. What displays would be needed for the effect to work, and be useful in which situations? It is known that synchronised visual and tactile stimulation is important. What of senses such as proprioception and audition? What types of sensors work best and in which situations? What motor possibilities are needed? 
Distributed embodiment can be accomplished in virtual reality, but it can also at least in principle - be implemented in the blended reality of the physical and the virtual that is increasingly our everyday habitat. Cameras and other sensors, mobile and large scale displays, physically close and distant people will interact through context-sensitive applications producing - as appropriate - altered, expanded and distributed embodiment.

\subsection{Conclusion to Chapter 2}

The notion of altered embodiment - that we can change our sensory and perceptual capacities in the physical, through the use of mediating information technology - is not unfamiliar to us. Take the example of regularly wearing corrective spectacles as an obvious example. But some of the possible applications and potential uses may be less familiar. Similarly, we are not unfamiliar with what we are calling expanded embodiment. Every interactive game player is used to having their attention transported to another place, as if their bodies were extended into the fictional game space. Similarly, we can readily understand that the controller of an American drone flying into foreign airspace has the experience of being in that place to some extent, and not where his or her physical body is actually located. Distributed embodiment is a stranger concept, since few people have experienced out-of-the-body experiences and, until recently, this was not recognised as a possibility for technological exploitation.

The idea of distributed cognition (Perry 2003) is familiar, along with a variety of related views about how information technologies are assimilated into the way we make sense of and function in the world (e.g., Clark 2003, Dourish 2001, Hutchins 1996, Kaptelinin \& Nardi 2006,). However, not all technology that we use for cognitive support becomes integrated with the self; the measurement of experienced presence allows us to distinguish when this happens and when it does not. If the technology is integrated with the self, we attend to and feel present in an external mediated reality in which the mediated nature of the world is invisible. The technology effectively becomes part of the body - we are embodied through the technology. As we have seen this can happen in a variety of ways. If the technology is not integrated with the self, using the technology involves a transfer of attention away from the mediated world and we feel absent from it. These feelings translate into measurable presence phenomena.

I may feel myself to be present here, in a state of altered embodiment; there, through expanded embodiment, and - potentially at least - as someone else there, via distributed embodiment. When I feel present here, in my usual body-centric mode of first-person observation of the world around me, I can alter my experience of the world in a wide variety of ways by technological means. This may be simply a matter of improved vision or hearing, or it could be more analogous to the effects of taking a psychotropic drug - a radically altered perception of the physical world of time and 
space surrounding my body. When I am said to be present there, this refers to my perception of a world around me that is not the physical world in which my body is actually located. I am virtually and experientially located there (Riva et al. 2002) not here. When I am someone else there, I experience presence there and yet also observe myself as another body. This is a new and puzzling form of interactive presence, with as yet unclear potential for the future of mediated embodiment.

Although three approaches to designing interactive presence have been described in this conceptual chapter, characterising three different forms of embodiment, they are not discrete categories. Perhaps most promising and most challenging is the design of new forms of embodiment in reality blends of the physical and virtual. Such blends are of unimaginable variety, but must be designed to meet the needs of actual people with needs and purposes in the social, shared and physical world in which we still all live and will die. Presence is the yardstick of embodiment from an experiential perspective. If you cannot feel presence, you are not embodied in the world.

\section{References}

Bach-y-Rita, P., \& Kercel, S. W. (2003). “Sensory substitution and the human-machine interface.”. Trends in Cognitive Neuroscience, 7(12), 541-546.

Benyon, D (2012). Presence in Blended Spaces. Interacting with Computers, 24(4), 219-226.

Blackmore, S. (1984). A psychological theory of the out-of-body experience. Journal of Parapsychology. 48, 201-218.

Bouchard, S, Dumoulin, S, Talbot, J, et al. (2012). Manipulating subjective realism and its impact on presence: Preliminary results on feasibility and neuroanatomical correlates. Interacting with Computers, 24(4), 227-236.

Biocca, F. (1997). The cyborg's dilemma: Progressive embodiment in virtual environments. Journal of Computer-Mediated Communications, 3(2).

Biocca, F. A., \& Rolland, J. P. (1998). Virtual eyes can rearrange your body: Adaptation to visual displacement in see-through, head-mounted displays. Presence, 7, 262- 277.

Botvinick, M., \& Cohen, J. (1998). Rubber hands 'feel' touch that the eye sees. Nature, 391, 756.

Bracken, C. \& Skalski, P. (2010) (Eds.) Immersed in Media: Telepresence in Everyday Life. New York: Routledge.

Castiello, U., Lusher, D., Burton, C., et al. (2004). Improving left hemispatial neglect using virtual reality. Neurology, 62, 1958-1962.

Clark, A. (2003). Natural Born Cyborgs: Minds, Technologies, and the Future of Human Intelligence. Oxford: Oxford University Press.

Dourish, P. (2001). Where the Action Is: The Foundations of Embodied Interaction. Cambridge, MA: MIT Press.

Ehrsson, H. H. (2007). The Experimental Induction of Out-of-Body Experiences. Science 317 (5841), 1048.

Ehrsson, H. H., \& Petkova, V. I. (2008). E-letter response to: H. H. Ehrsson (2007). The experimental induction of out-of-body experiences, Science, 317, 1048.

Floridi, L. (2004). Exploring the informational nature of presence. Opening invited keynote address at 7th Annual International Workshop on Presence. Polytechnic University of Valencia, Spain, 13-15 October 2004. 
Google Glass (2013). http://www.google.com/glass/start/. Accessed November 7, 2013.

Gregory, R. L. 1997. Eye and Brain. 5th edition. Oxford: Oxford University Press.

Haans, A., \& IJsselsteijn, W. A. (2012). Embodiment and telepresence: Toward a comprehensive theoretical framework. Interacting with Computers, 24(4), 211-218.

Hochberg, L. R., Serruya, M. D., Friehs, G. M. et al. (2006). Neuronal Ensemble Control of Prosthetic Devices by a Human with Tetraplegia. Nature 442 (7099),164-171.

Holmes N., \& C. Spence (2007). Dissociating Body Image and Body Schema with Rubber Hands. Behavioral and Brain Sciences 30, 211-212.

Hoshi, K., \& Waterworth, J. A. (2009). Tangible Presence in Blended Reality Space, PRESENCE 2009. Proceedings of the 12th Annual International Workshop on Presence. November 11-13, Los Angeles, CA, USA.

Hoshi, K., Öberg, F. \& Nyberg, A. (2011) Designing blended reality space: conceptual foundations and applications,

Proceedings of BCS-HCI '11: the 25th BCS Conference on Human-Computer Interaction, 217-226.

Hutchins, E. (1996). Cognition in the Wild. Cambridge, MA: Bradford Books, MIT Press.

Ihde, D. (1990). Technology and the lifeworld-From garden to earth. Bloomington and Indianapolis: Indiana University Press.

IJsselsteijn, W. A. (2005). Towards a neuropsychological basis of presence. Annual Review of CyberTherapy and Telemedicine, 3, 25-30.

IJsselsteijn, W.A., de Kort, Y.A.W., \& Haans, A. (2006). Is this my hand I see before me? The Rubber Hand Illusion in Reality, Virtual Reality, and Mixed Reality. Presence: Teleoperators and Virtual Environments 15, 455-464.

Kaptelinin, V., \& Nardi, B. (2006). Acting with Technology: Activity Theory and Interaction Design. Cambridge, MA: MIT Press.

Larsson, M., D. Larhammarb, M. Fredrikson, P. et al. (2005). Reply to M. A. Persinger and S. A. Koren's Response to Granqvist et al. Sensed Presence and Mystical Experiences Are Predicted by Suggestibility, Not by the Application Of Transcranial Weak Magnetic Fields. Neuroscience Letters 380 (3), 348-350.

Leeb, R., D. Friedman, G. R. Müller-Putz, R. et al. (2007). Self-Paced (Asynchronous) BCI Control of a Wheelchair in Virtual Environments: A Case Study with a Tetraplegic. Computational Intelligence and Neuroscience, 20 (April 2007): Article 7.

Lenggenhager, B., Tadi, T., Metzinger, T. et al. (2007). Video Ergo Sum: Manipulating Bodily SelfConsciousness. Science 317 (5841), 1096-1099.

Lombard, M., \& Ditton, T. (1997). At the Heart of It All: The Concept of Presence. Journal of Computer Mediated-Communication 3(2).

Merleau-Ponty, M. (1962). The Phenomenology of perception. Translated by Colin Smith. London: Routledge and Keegan Paul.

Metzinger, T. (2006). Reply to Gallagher: Different conceptions of embodiment, Psyche 12(4).

Minsky, M. (1980). Telepresence. Omni Magazine, June 1980.

Misselhorn, C. (2009). Empathy with Inanimate Objects and the Uncanny Valley. Minds and Machines 19 (3), 345-359.

MIT Project Oxygen. Computer Science and Artificial Intelligence Laboratory. http://www.oxygen.Ics. mit.edu/. Accessed 7 November 2013.

Nagel, T. (1974). What Is It Like to Be a Bat? Philosophical Review 83(4), 435-450.

New Scientist (2010). The Real Avatar: Body Transfer Turns Men into Girls. May 13. http://www. newscientist.com/article/dn18896-the-real-avatar-body-transfer-turns-men-into-girls.html. Accessed June 27, 2013.

Norman, D. A. (1993). Things that Make Us Smart. Reading, Mass: Addison-Wesley.

Normand, J.-M., Giannopoulos, E. Spanlang, E. B et al. (2011). Multisensory Stimulation Can Induce an Illusion of Larger Belly Size in Immersive Virtual Reality. PLoS ONE 6(1): e16128. 
Perry, M. (2003). Distributed Cognition. In J. M. Carroll, (Ed.) HCI Models, Theories, and Frameworks: Toward an Interdisciplinary Science, (193-223). San Francisco, CA: Morgan Kaufmann.

Persinger, M. A.,. Saroka, K., Koren, S. A. et al. (2010). The Electromagnetic Induction of Mystical and Altered States within the Laboratory. Journal of Consciousness Exploration \& Research 1(7), 808-830.

Petkova V.I. \& Ehrsson H. H. (2008). If I Were You: Perceptual Illusion of Body Swapping. PLoS ONE 3(12): e3832.

Riva G. (1998). Modifications of body-image induced by virtual reality. Perceptual \& Motor Skills, 86(1), 163-70.

Riva, G. (2007). Virtual Reality and Telepresence. Science, 318(5854), 1240-1242.

Riva G., Davide, F \& IJsselsteijn, W.I. (2002). Being There: Concepts, Effects and Measurements of User Presence in Synthetic Environments. Amsterdam: IOS Press.

Riva, G., Waterworth, J. A. and Waterworth, E. L. (2004). The Layers of Presence: a bio-cultural approach to understanding presence in natural and mediated environments. Cyberpsychology and Behavior, 7(4), 402-416.

Riva, G., Waterworth, J. A., Waterworth, E. L. et al. (2011). From Intention to Action: The Role of Presence. New Ideas in Psychology 29(1), 24-37.

Slater, M. \& Steed, A.J. (2000). A virtual presence counter, Presence: Teleoperators and Virtual Environments, 9(5), 413-434.

Slater M., Perez-Marcos, D. Ehrsson, H. H. et al. (2008). Towards a Digital Body: The Virtual Arm Illusion. Frontiers in Human Neuroscience. 2, Article 6, 1-8.

Slater, M., Spanlang, B. Sanchez-Vives, M. V. et al. (2010). First Person Experience of Body Transfer in Virtual Reality. PLOS ONE 5(5): e10564.

Slotema, C. W.,. Blom, J. D., Hoek, H. W. et al. (2010). Should We Expand the Toolbox of Psychiatric Treatment Methods to Include Repetitive Transcranial Magnetic Stimulation (rTMS)? Journal of Clinical Psychiatry 71(7), 873-884.

The vOICe (2014). http://www.seeingwithsound.com. Accessed 30 January 2014.

Waterworth, E. L. \& Waterworth J. A. (2001). Focus, Locus and Sensus: the 3 Dimensions of Virtual Experience. Cyberpsychology and Behavior, 4(2), 203-214.

Waterworth, J. A. (1997). Creativity and Sensation: The Case for Synaesthetic Media. Leonardo, $30(4), 327-330$.

Waterworth, J. A. (2003). Virtual Realisation: Supporting Creative Outcomes in Medicine and Music. Psychnology, 1(4), 410-427.

Waterworth, J. A. and Fällman, D .(2003). The Reality Helmet: Transforming the Experience of Beingin-the-World. Proceedings of HCl 2003: Designing for Society, Bath, UK, Volume 2, 191-194.

Waterworth, J. A .\& Waterworth, E. L. (2003). The Core of Presence: Presence as Perceptual Illusion. Presence-Connect, 3(3), posted 24-07-2003.

Waterworth, J. A., Waterworth E. L., Mantovani, F. et al. (2010). On feeling (the) present: an evolutionary account of the sense of presence in physical and electronically-mediated environments. Journal of Consciousness Studies, 17(1-2), 167-189.

Yee, N. \& Bailenson, J.N. (2007). The Proteus Effect: Self transformations in virtual reality. Human Communication Research, 33, 271-290. 